\title{
Common data and technological partnership - the foundation for the development of smart cities - Poznań Case Study
}

\author{
Cezary Mazurek \\ Poznan Supercomputing \\ and Networking Center, Poland \\ mazurek@man.poznan.pl
}

\author{
Maciej Stroinski \\ Poznan Supercomputing \\ and Networking Center, Poland \\ stroins@man.poznan.pl
}

\begin{abstract}
Over the recent years communities have been working towards changing the paradigm of city development into the so-called smart approaches. While various revolutionary solutions have been deployed to make the cities smarter, we believe that a more evolutionary path makes it easier for the cities to change into smart ecosystems. Such an evolutionary path is possible with the right foundation. In this paper we discuss such a foundation that has been making the city of Poznan, Poland, smarter over the last 20 years, and opens opportunities for employing the Citizen Science model of smart city development. This foundation relates to the combination of the creation of a common data space, and the technological partnership with a research and development center and research cyberinfrastructure operator such as the Poznań Supercomputing and Networking Center.
\end{abstract}

\section{Introduction}

In the age of Data Science and its challenges, urban development related to the idea of creating Smart Cities, covers an ever wider range of life of the inhabitants and administration and reveals new aspects of the evolution of these ecosystems. Managing an intelligent city is impossible today without taking into account such horizontal aspects as: openness of data, infrastructure available always and everywhere, the Internet of things [1]. They intersect all vertical aspects of urban life such as citizen participation, health and social well-being, safety, creativity and arts, education and culture, etc. and through this intersection affect the scale of smart applications.

In relation to these places, the development of technologies and smart applications takes place most often through the implementation of projects, which with the use of the so-called Proof of concept method verify the usefulness of technologies or concepts of application scenarios. The availability of advanced cyber infrastructures, including modern IoT platforms, is an essential element in the implementation of such projects. Moreover, the implementation of them is placed in a real urban environment and their effects are more embedded in the life of the city, its inhabitants and its administration. Examples of such projects include areas such as safety [2,3], transport [4,5], air quality [6,7], health and wellbeing [8,9], senior policy [10], etc. The application of emergent technologies in these areas leads to the creation of new aspects of the functioning of the city and its ecosystem, just as the application of disruptive technologies in other areas of life influences the direction of their evolution, and often promoting them very quickly to an unexpected level of modernity (the so-called digital vortex effect [12]).

A separate issue important from the point of view of stability and continuous functioning of complex ecosystems such as a city, is integration with the existing and functioning systems in the above mentioned processes. This problem often requires the deployment of dedicated solutions, the development of which requires strong competences in such technological areas as: interoperability and integration of IT systems, efficient processing of large amounts of data, advanced distributed processing architectures, agile programming methods, etc.

In the article we will present a case of building a smart city system in Poznań, which illustrates the factors that allowed for the evolution towards a real smart city ecosystem. In chapter 2 we will present Poznan in the context of developing a smart city. The third chapter is devoted to the presentation of the MIM information platform, which has been operating in the city for over 20 years. Chapter 4 presents selected infrastructure and dedicated application areas. Chapter 5 presents the directions of integration from the point of view of Smart City and the last chapter - a new vision re-created according to the Citizen Science model. 


\section{Developing a Smart City of Poznań}

Poznań, with a population of 540 thousand (data from 2016), is the capital of the Wielkopolska region, an important academic center (8 universities, 33 research institutes), cultural centre (4 theatres, opera house, philharmonic hall) and an important economic center with easy access to advanced IT infrastructure. Among the region's smart specializations, which are all strongly linked to modern technologies, are the following: ICT-based development, Modern medical technologies, Specialized logistics processes, Manufacturing industry of the future, Biomaterials and food and Interiors of the future [13].

The Poznan Supercomputing and Networking Centre (PSNC) was established in 1993 and affiliated to the Institute of Bioorganic Chemistry Polish Academy of Sciences with the mission to develop the IT infrastructure for science and conduct $R \& D$ in the scope of advancement and applications of digital technologies (in Europe referred to as ICT Information and Communication Technologies). At the end of the 20th century, Europe created a vision of building the Information Society, which ended with the adoption of the information society strategy and an Action Plan entitled "Europe's Way towards the Information Society, in 1994 [14]. The ideas of this strategy became an impulse for establishing cooperation between the Poznań City Hall and PSNC in the field of building pilot solutions for the information society. Initially, the cooperation concerned mainly two areas: cooperation in the field of building a fiber-optic network as a basis for creating broadband application solutions of the future and building a Municipal Multimedia Guide (MIM) as a communication platform between the city and its inhabitants and visitors [15]. The cooperation in the field of building a fiber-optic network resulted in the connection of distributed branches of the City Hall to the scientific metropolitan are fiber-optic network. Cooperation on the MIM has enabled a thorough understanding of the local ecosystem and the identification of local needs. As a result, the city began to use IT technologies in order to increase the efficiency of its local infrastructure (towards smart city), as well as to cooperate and raise the awareness of its citizens (towards citizen science).

It is worth noting that at the same time the City Hall implemented informatization of the office, which was separate from the cooperation with PSNC. However, it was difficult to avoid completely the need to link some activities. The effect of linking the activities of the City Hall services with the MIM platform was to build a network of source guides for this platform, and currently more than 2500 employees of the City Hall are responsible for the substantive editing of information. Mutual relations also appeared in the creation of new services for citizens, e.g. the first in Poland electronic service of the Business Activity Register (2004), the system of supporting recruitment to schools Recruitment (completed in Poznań as early as 2003), access to the certificates of the registry office. They were the first reference websites available in Poland. As a result, the company received awards in national competitions and rankings, including the IT Leader (2004), - Euro Crest Awa@rd the best website (2006, 2011).

In 2011, Poznan was the first city in Poland to publish a pilot set of application interfaces (APIs) to provide, among other things, selected subsets of spatial data, data on events in the city, on the MIM platform. The data sets were made available on the basis of the principles set out in the Act on Access to Public Information and similar to the conditions of the Creative Commons Attribution-Share Alike 3.0 license. As part of the promotion of this undertaking, Poznan organized the first informal workshops for programmers (hackaton), consisting of joint work on the data made available. Subsequent workshops helped to shorten the relationship between decision-makers and society.

Global development trends in relation to cities, as well as experience in cooperation with PSNC, led to the formulation of the City Development Strategy to 2030 [16] which includes following priorities (Figure 1):

- Strong Metropolis,

- Smart entrepreneurship,

- Green, mobile city,

- Friendly, residential estates,

- Community and social dialogue.

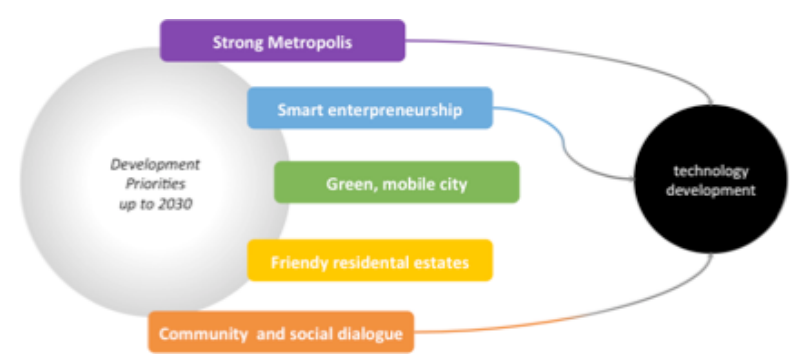

Figure 1. Development priorities for City of Poznan

In 2017, the PSNC was selected as the official technological partner of the City Hall in the area of digitization and at the same time a plenipotentiary for Smart City was appointed by the Mayor of Poznan. His 
tasks include, among others, implementation on its own initiative and coordination of solutions related to the smart city, providing opinions on projects, initiatives and undertakings of strategic nature for the city of Poznań, taking into account new information and communication technologies, providing opinions on projects and initiatives, preparation of principles of data openness policy and extending the areas of data and content sharing belonging to the city of Poznan. The position will facilitate the management of projects for the development of applications with the participation of decision-makers (joint analysis and adaptation of processes) and the development of citizen science on the current MIM platform.

In this situation, cooperation models operating in the scientific community, where advanced IT infrastructure (Cyberinfrastructure) and evolutionary model of cooperation based on open access to data, in the present era are an interesting and effective alternative, constitute the strength of development of a given field and the scale of the challenges to be addressed. Therefore, adaptation of cooperation models implemented in virtual environment (e.g. based on the concept of Science Gateways) can leverage smart applications platforms to citizen science ecosystem in an evolutionary way.

\section{MIM Information Platform}

The result of cooperation between PSNC and City Hall is an electronic and service platform, serving an average of nearly 6 million users per year, collecting over $210 \mathrm{~GB}$ of data. Using dedicated editorial services, officials of the City Hall and its subsidiaries continuously manage information (including BIP Public Information Bulletin), submitted by more than 2500 substantive editors and replicated from the City's internal systems. The platform is distinguished by the mechanisms of content and service sharing. More than 20 REST collections, nearly 700 categories and nearly eight thousand POI present geolocalized content, events and news. Access is provided in the open data model, through dedicated application interfaces (APIs) for external application providers - Poznań is a pioneer in this respect in Poland, nearly 20 applications have been created on the basis of open APIs to date. Part of the data from MIM is also replicated to the government platform danepubliczne.gov.pl - Poznan makes available 25 sets of data, mainly from the BIP of the City of Poznan.

The platform provides a number of e-services for residents, tourists and officials, including a digital plan based on reference information resources, a platform for public consultations, a platform for civil budget services (about 45,000 applications in 2017), air quality, Internet transmissions from the City Council (on average 100 people observing/session of the City Council), Internet Assistant Councilor, Internet Assistant President, "Interventions-Poznan" (reporting interventions to city services from mobile devices nearly 7.5 thousand notifications in 2017, over 3.2 thousand application users) or SmartZOO - a mobile assistant for visitors in the form of an intelligent multimedia recreational and educational space (over 7 thousand application users).

Pilot access to application interfaces implied the creation of a service describing the API. Detailed technical information is available at https://egov.psnc.pl/api/ Information on this website is intended mainly for programmers.

The applied architecture of the MIM platform, thanks to its original approach, enabled incremental adding of new data sets, creation of services and smart apps.

Over time and with the development of resources, the scope of content was expanding. A major qualitative leap was the launch of the open API of the Poznań BIP. Nowadays, thanks to this API it is possible to reuse the content and data from several dozen databases automatically, without the need to submit any applications.

Great importance was attached to opening APIs developed within the cognan.pl system of services and applications. It was assumed that inhabitants using the market offer of applications and services (e.g. mobile) should not be condemned to installing urban solutions, but should be able to supplement them with services compatible with the urban system, but provided by self-selected suppliers.

The last element, which is currently being developed within the city platform, is data and content collections obtained from the City Hall of Poznan, Municipal Organizational Units and cooperating institutions, which have not yet been equipped with automatic interfaces for sharing, but their substantive value can be useful thanks to their reuse in building services and studies for the residents by external users.

The currently available data sets will be made available through a dedicated platform based on the Comprehensive Knowledge Archive Network (CKAN), which is becoming the reference framework for publishing open data in one place. This system would complement the MIM platform and the CKAN system would be a typical data hub system.

The vast amount of data available on the platform leverages the creation of new smart applications. Advanced e-Infrastructure (or Cyberinfrastructure) is the foundation of this development. 


\section{From Data through Infrastructure to Smart Applications}

\subsection{Infrastructure}

Creating Smart City is not possible without wisely expanding the urban infrastructure with devices that will become a source of data for further services. Examples of such infrastructure include Intelligent Transport System (ITS), Poznań City Bicycle (PRM), car rental for minutes (external operators). Each of these systems shall provide data that can be consumed in a variety of ways (Figure 2).

In the city space there are currently many elements of information infrastructure that mobilize the city to develop smart applications with multiple threads. Several of these can be mentioned.

4.1.1 Fiber optic network infrastructure is composed of two networks: the metropolitan area scientific network POZMAN and the network connecting the city's monitoring cameras. The POZMAN network in the area of the Poznan agglomeration has $110 \mathrm{~km}$ of own fiber optic cables connecting all scientific units of the city of Poznan and some schools. PSNC, as the operator of the POZMAN network, also provides a corporate network of the Office, connecting many city units and a wireless network, on the basis of which free access to the Internet is offered. The fiber optic network for connecting cameras is being built by the Crisis Department of the City Hall. There are interrelationships between networks including exchange of fibers, pipes as well as co-investments.

4.1.2 Wireless@Poznan Zone is using the 802.11 standard and mesh topology whereas access network enables $802.11 \mathrm{~b} / \mathrm{g}$ standards and was built by the city of Poznan in cooperation with and managed by PSNC. It covers the city center and several major commuting routes, including the main railway station.

4.1.3 CCTV system in the city is a system of cameras based on own optical fibers operated by the police. The data obtained from the cameras is stored for the period required by law. There is currently no access to this data outside of the authorized services. It is possible to gain access to $R \& D$ projects implementing applications of data analysis and data contexts. PSNC implemented one of such projects.

4.1.4 ITS - Intelligent Transport System is an ICT infrastructure under development by relevant agencies of the city of Poznań, aimed to optimize the city transportation system through real-time monitoring of vehicles, parking spaces and traffic, and provision of the collected data to other, external systems. Some of the envisaged applications include real-time information on the public transportation (e.g. at bus/tram stops), estimation of driving time in the context of current traffic, provision of information on parking spaces availability and prioritizing public transportation.

4.1.5 Community Traffic Online is an Urban navigation system developed and marketed by NaviExpert, providing a number of personalized services enabling to navigate through the urban traffic. It seamlessly integrates traditional navigation information with easily submitted reports from the user community (moving in cars, on bikes and on feet) delivering context-based routes. The system won the traffic navigation competition in the city of Warsaw in 2012.

4.1.6 PEKA - Poznan Agglomeration Electronic Card is a system managing electronic cards for the residents of Poznan, which is aimed at unifying the way of consuming transportation services delivered by different providers and enables to benefit from various services such as parking zone ticketing, library usage, sports, e-wallet, e-signature and e-identification. It is foreseen that the system is connected with real time monitoring of public transportation vehicles. First functionalities of PEKA have been in place in 2013.

\subsubsection{Ambient Assistant Living installation} developed by PSNC within the HELPS project (cofinanced by the Central Europe program - www.helpsproject.eu), it creates a network of 15 smart homes (real homes of elderly and disabled persons) built using the universAAL platform and providing several services and applications. Services and applications are adapted and deployed in cooperation with the Centre for Senior Citizen Initiatives (public municipal agency with the mission of activating the elderly population of the city) and non government organizations.

4.1.8 City GIS developed by PSNC based on open source software and integrating large amount of geospatial information from distributed systems, it is used by the city of Poznan within its e-services platform for citizens (http://www.poznan.pl/plan/). It allows to place virtual objects on a digital map, binding geographical location and meta-definition of objects. The data collected in the system is available for use within the open data paradigm. 


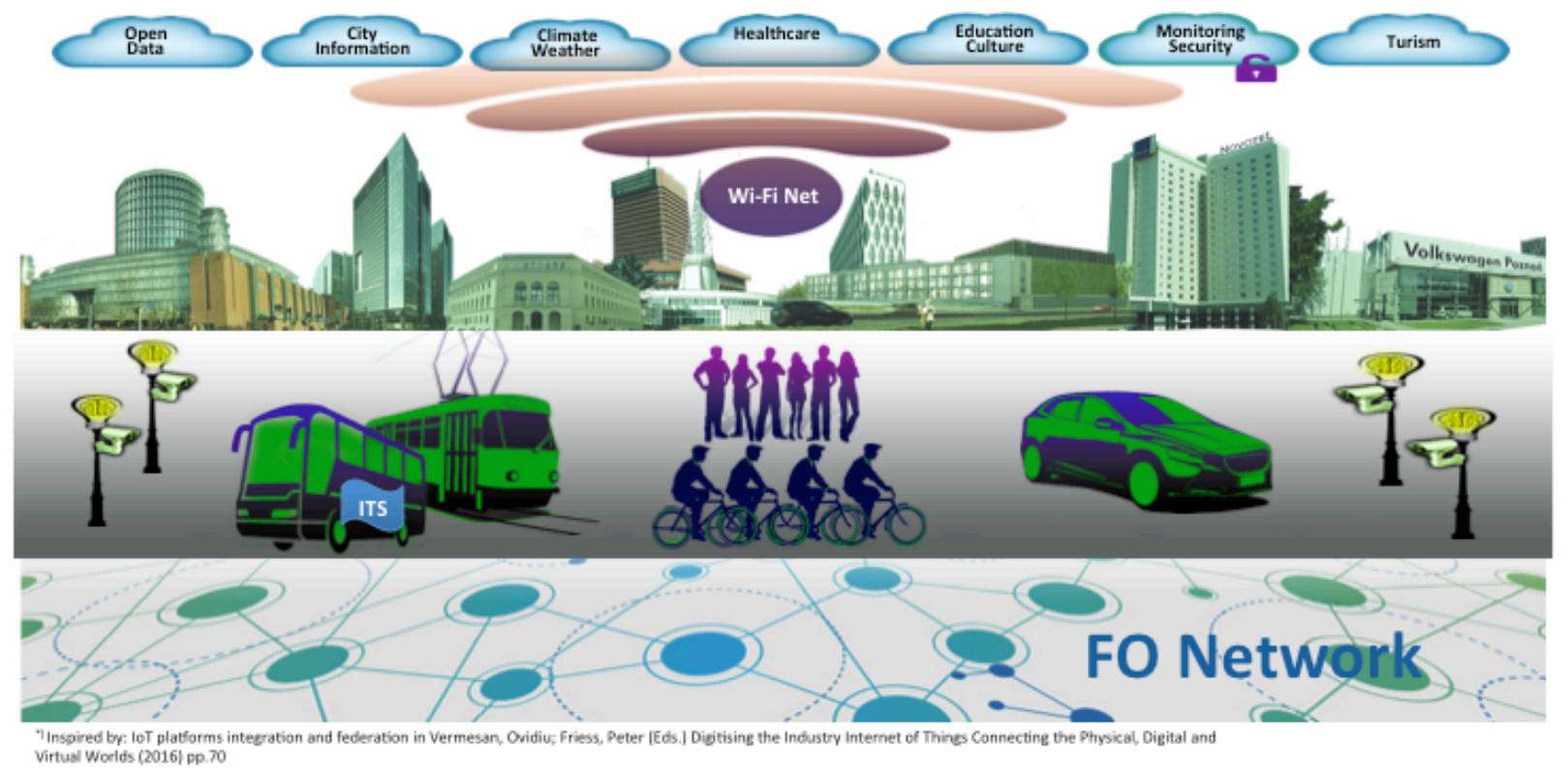

Figure 2. Smart City and Smart Applications

\subsection{Applications}

The data provided and the infrastructure allow you to create IT applications. The City of Poznań, together with PSNC, made such applications available to the residents. These include, but are not limited to:

SmartZOO personal mobile guide and assistant to the visitor of ZOO Park, providing attractive, interactive educational content - before, during and after the visit.

Atmosphere for Poznań - information on the forecasted and actual condition of air quality in Poznań, related to the applicable legal regulations in Poland - the detection of unfavorable conditions is automatically connected with the decision on free public transport, communicated in the application.

Plan of the City of Poznan - simple visualization of the spatial data held and made available.

Poznań Addresses - the application contains a database of over two thousand streets and almost 60 thousand addresses of the City of Poznań. Allows you to find a specific address, all addresses for the selected street or near the location indicated on the map.

When I go - PEKA - the application informs about the exact time of departure of the bus or tram. The data comes from the ITS system, installed e.g. in public transport vehicles or at bus stops, and is updated on an ongoing basis, taking into account the current traffic situation.

Mobile Poznan - the application includes a timetable monitor with a search engine for stops and their visualization on the map, an interactive map of ticket vending machines and parking lots. The whole event was supplemented with news and announcements of cultural events and events.

Poznań news - application presenting information from RSS feeds coming from Poznań's websites.

Poznań Guide - application developed during the first hackathon of the API poznan.pl. It allows you to find the place you are looking for. It can be a cinema, an MPK bus stop, a hotel, a restaurant, a pub, a club, a 24-hour shop and many more. Using the application Poznań Guide you can determine your location and map your route to each of these places.

Sacral Poznan - the Sacral Poznan application was created during the hackathon API Poznan.pl with the thought of people planning to visit the graves of their loved ones in mind. Its purpose is to make it easier to find a burial place for specific people and to search for cemeteries and churches in Poznań.

Full list of applications is available on the website of the City of Poznan.

\section{The most important directions of integration from the point of view of Smart City}

The infrastructure, applications and MIM information platform discussed above constitute a solid technological basis for building the Intelligent City of the Future. The adoption of the Poznan 2030 strategy for implementation with clearly indicated priorities requiring absolutely intelligent solutions, which will make the city innovative and offer a high quality of life 
in effective management procedures and services, is a political declaration of going down this road. This is confirmed by the creation of dedicated positions in the city management structures related to the implementation of the vision of the Intelligent City, as well as technological partnership with a recognized scientific unit (i.e. PSNC).

The transition to the Smart City based on Open and Citizen Science paradigms requires the following to be met [17]:

- Content and data resources shall be publicly available;

- The administration does not have a monopoly on the best solutions of information systems implemented thanks to new technologies;

- You know best (or should know best) how to use public domain information;

- The "smart city" community cannot be built without active cooperation based on the "dialogue of equals" a conversation based on access to the same knowledge

- Additionally, the data are made available to all interested users, i.e. to the public:

- Residents and groups of Residents (e.g. organised in the Housing Estates Council, Association, NGO...)

- Creators of websites, mobile applications, new eservices

- SMEs: encourage the use of resources to build their business offer to citizens

As a result of the synergy - the data provided will support the platform and the competences of the plenipotentiary - cooperation opportunities will be created at the level of creating small-scale projects, which will enable the creation of advanced services in the areas of machine learning, bid data or visualization of the data held. An example of this is the visualization of budget data or the use/coverage of city bikes in the context of events in Poznań.

An additional context for integration is provided by the ICT R\&D strategy referred to in Europe as Next Generation Internet - a key priority in H2020, which will ensure a more human-centric Internet supporting European values of openness, cooperation across borders, decentralisation, inclusiveness, transparency and protection of privacy. The NGI initiative is a broad and long-term initiative, mostly focusing on challenges related to new technologies as for example a better protection for private data, new Internet architectures, decentralized data systems and new discovery and identification tools. These technologies, developed by people and for people, will widen our space for life and work in future Europe.

In this context, it is important to mention the barriers that are today challenges to the development of real Citizen Science ecosystems. The most important of these are:

- Reliability of data

- Access to scientific and commercial data

- Interoperability of data sets

- Ownership and IPR for data and results

- Privacy and anonymization techniques

- Software - openness and modularity

- Cultural and linguistic diversity

- $\quad$ Security

Thinking about Citizen Science in the category of the ecosystem, we can create conditions for overcoming these barriers through coordinated actions in the areas of: local government, science and technology as well as social.

Due to the relatively high level of readiness of the city to transform into a smart city of the future, it seems that it should be an evolutionary one. However, this is a very dangerous situation. On the one hand, not all systems operating in the city are able to provide data in the form required to implement integration processes, which in extremely difficult situations may require the exchange of these systems, and on the other hand, further development must take into account natural technological revolution identified by the visions of the NGI (Figure 3) or described by the availability of so-called Disruptive Technologies, e.g.: Internet of Things, 5G networks, Computational Clouds, Big Data, Machine Learning and Artificial Intelligence, Virtual and Augmented Reality, Advanced Visualization.

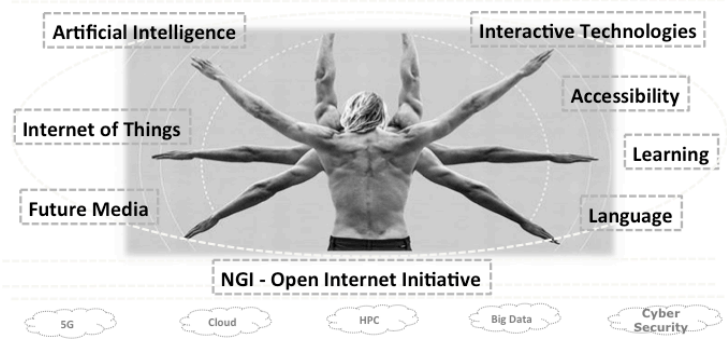

Figure 3. Next Generation Internet ${ }^{1}$

Totally "infecting" the life processes of the city and the lives of people in the city with digital technologies, despite the undisputed usefulness of the implemented solutions will be associated with the need to solve security problems and to create trust in the technology.

Digitalization of the city towards Smart City is a great social and economic challenge, so success is possible only with active involvement of the inhabitants in these processes. Therefore, the

\footnotetext{
1 Based on https://www.ngi.eu/resources/ec-calls/
} 
participation of residents in research, creation of a "proof of concept", verification of new solutions and implementations is mandatory.

The instruments that are used to implement this participation are Living Laboratories and Coworking Space, which operate at PSNC in the so-called FutureLab space [18]. Living laboratories operate in the areas of Education and Culture, Health and Quality of Life, Digitization and New Media. Coworking space participants have the opportunity to use in their work access to technological PSNC laboratories, including access to cloud and HPC computing. Therefore, it is possible to carry out pre-incubation processes of innovative solutions, for example, hackatons (one of which was mentioned earlier). As a result of three years of Coworking Space activity, 10 startups were created.

\section{A new vision based on the Citizen Science model}

\subsection{Smart City innovation areas}

As discussed above, building a smart city is a complex ecosystem in which residents play an important role in the concept of Citizen Science. Their activity can be seen in different categories: [19]:

- Collaborative Science,

- Collective Intelligence,

- Participatory experiments,

- Crowd-crafting,

- Volunteer thinking,

- Human sensing,

- Volunteer sensing,

- Volunteer computing.

Smart City innovation areas are most often associated with: Open Data, City Information, Climate and Weather, Healthcare, Monitoring and Security, Education and Culture, Tourism etc.

Therefore, the question arises of how in particular areas the activities of the inhabitants within the framework of the Citizen Science can be connected with individual areas. The prerequisite for the operation of this system is the existing and wellfunctioning infrastructure and applications (Fig. 4)

With regard to Smart City in Poznań, we can illustrate these links in three exemplary areas of innovation: Climate/Weather, Education/Culture, Open Data.

\subsection{Climate and Weather innovation area}

One example within the area of Climate and Weather is the application of the Air Quality
Observatory. It will operate in an environment of data provided by state services as well as residents and simulation tools provided by research institution.

The State Services have certified data collection stations according to standardized procedures. Some of these stations are automatic, but their number and location are limited. Some services are built on this data, such as the one mentioned in 4.2 (Atmosphere for Poznań). At the same time, independent social services provide data from privately operated stations. These stations are cheap, widely available but not certified. The data from this observatory can be used as input to a system of multiscale air quality simulation in the areas of quarters, districts or the whole city, with changing conditions and meteorological forecasts [20].

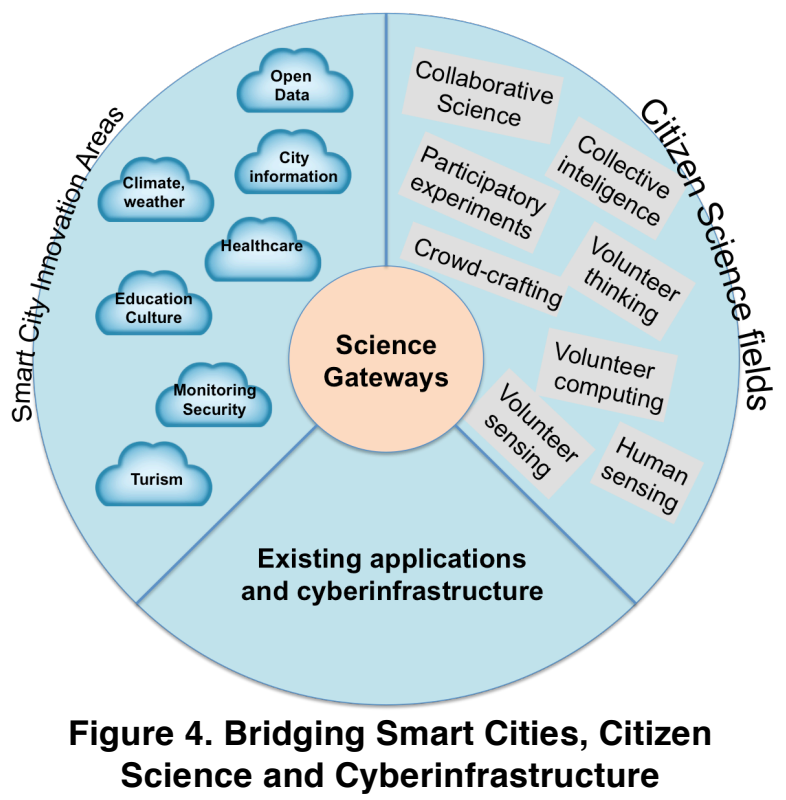

Such a system, using the reverse modeling method, may help to identify the sources of contamination. Government institutions, society and science are involved in its construction. From the point of view of building a global observatory for a smart city, we should have a common point of concentration of data and activities of these groups. Such a point can be well organized and maintained according to the idea of the Science Gateway. It can therefore have a long-term impact on each of these groups: it broadens the experiment for learning, the data for government and a comprehensive knowledge for the citizens. The existence of such reliable information may also provide the basis for the development of further innovative services, such as: informing the residents of asthma patients about the occurrence of unfavorable conditions, locating places where the well-being is 
worse or negative symptoms are felt, which is directly related to the Health innovation area.

\subsection{Education and Culture innovation area}

In the area of education and culture, an example of multidimensional activities is the digitization of cultural heritage resources held by citizens in relation to a given region. The reference to local and regional cultural heritage is important from the point of view of smart cities. Digital resources related to this heritage are found in state institutions (e.g. the archives of State Archive, Public Television, Public Libraries as well as Scientific Libraries). Some of this material is already in digital libraries [21] others need to be digitized. An important aspect is to link these resources to private collections in society, the digitization of which is a challenge in its own. This challenge can be met by setting up a system of participatory digitization. The PSNC plans this in conjunction with the digitization laboratory and the Living Lab space at its disposal. In addition, we will use digitization tools (dINGO) [22] and digital libraries based on dLibra system [23]. Similar collections of social thematic digitization are organized by Europeana [24] and in this case, thanks to the existing infrastructure of regional digital libraries, private collections are kept locally. The combination of all the resources of the first collection and tools under the umbrella of Science Gateway will constitute an additional quality, using the mutual relations.

The available regional resources, collected in a comprehensive way, may constitute the basis for innovative applications serving targeted tourism, inspiration for field games and creative industries. In addition, they provide an extremely valuable research base in the field of digital humanities.

\subsection{Open Data innovation area}

In both of the above examples, data quality and reliability are very important. This problem exists in general for all open data in the context of a smart city. The main challenge in this respect is the reliability and quality of data in the context of Citizen Science.

Despite, there are many examples of key success factors for citizen science projects from the point of view of data trust it is a common issue for some scientific areas [19]. Crucial factors for many of these approaches are the quantification of uncertainty and the validation processes of data by reliable institutions. These components are necessary when important decisions have to be made in order to yield the desired results that depend on the uncertainty pertaining to both the datasets and the models behind them.
In this context, the cooperation model presented above and the MIM information platform already have the necessary features to validate data in the Citizen Science environment. The result of the cooperation is a model of data validation, which is in line with the approaches indicated in Science Gateways or Virtual Research Environments [25]. Applications, that are built on these data (such as those indicated in point 4.2, e.g.: When I go - PEKA, Mobile Poznań), function within the city structure and its information platform, which is a consequence of the same approach. Moreover, our system is connected with the functionality of open access to data, which was described earlier. It opens up the possibility of introducing data validation processes at several levels, starting from the structure itself, through collection and processing workflows, to the authorization profiles at the application level (including social networks mechanisms).

\section{Conclusions}

The cooperative cyberinfrastructure, city information platform - MIM and smart applications created a potential for building common data. The effect was achieved thanks to the permanent cooperation of the city of Poznan with the scientific institution (i.e. PSNC).

In the article we discussed the experience related to the construction of the smart city ecosystem, which through the appropriate approach and evolutionary development remains open to development according to the Citizen Science model. The foundations of the ecosystem that has been developed in Poznań for over 20 years are: common data space and technology partners with the research and development center, which is also the operator of the cyberinfrastructure. To sum up, the development towards smart city in the cities that have such a base can follow an evolutionary path even if revolutionary technologies are incorporated into smart applications.

Without the citizens there can be no change to the Citizen Science model. Science Gateways is a very important bridge in this effort. This link creates a new quality for science - it broadens the source base, enables changes in research methods, and leads to the formulation of new scientific theses. With the use of new technologies, it is possible to obtain completely new results of scientific research.

In such a model, the use of technology in the area of Smart City brings great practical benefits as well as exploratory perspectives for science, which is a driving force for technology development. 


\section{References}

[1] Catlett CE, Beckman PH, Tolva J, Boxall A, Haque U, James P, et al. Instrumenting the City. 2015.

[2] RERUM, REliable, Resilient and secUre IoT for sMart city applications. Website: https://ict-rerum.eu/

[3] Elmaghraby AS, Losavio MM. Cyber security challenges in Smart Cities: Safety, security and privacy. Journal of advanced research. 2014 Jul $1 ; 5(4): 491-7$

[4] REMOURBAN, REgeneration MOdel for accelerating the smart URBAN transformation. Website: http://www.remourban.eu/

[5] Menouar H, Guvenc I, Akkaya K, Uluagac AS, Kadri A, Tuncer A. UAV-enabled intelligent transportation systems for the smart city: Applications and challenges. IEEE Communications Magazine. 2017 Mar;55(3):22-8.

[6] DEEDS, DialoguE on European Decarbonisation Strategies, Website: https://deeds.eu/

[7] Chapman L, Muller CL, Young DT, Warren EL, Grimmond CS, Cai XM, Ferranti EJ. The Birmingham urban climate laboratory: An open meteorological test bed and challenges of the Smart City. Bulletin of the American Meteorological Society. 2015 Sep;96(9):1545-60.

[8] C3 Cloud: A Federated Collaborative Care Cure Cloud Architecture for Addressing the Needs of Multi-morbidity and Managing Poly-pharmacy. Website: http://www.c3cloud.eu/

[9] Pramanik MI, Lau RY, Demirkan H, Azad MA. Smart health: Big data enabled health paradigm within smart cities. Expert Systems with Applications. 2017 Nov 30;87:370-83.

[10] City4Age, Elderly-friendly City services for active and healthy ageing. Website: http://www.city4ageinsight.org

[11] Bryant N, Spencer N, King A, Crooks P, Deakin J, Young S. IoT and smart city services to support independence and wellbeing of older people. InSoftware, Telecommunications and Computer Networks (SoftCOM), 2017 25th International Conference on 2017 Sep 21 (pp. 16). IEEE.

[12] Public Sector and the Digital Vortex, How Government Organizations Can Harness Digital Disruption for Positive Change.Global Center for Digital Business Transforamtion, June 2017
[13]

http://s3platform.jrc.ec.europa.eu/regions/PL41/tags/PL41

[14] Europe's way to the information society: an action plan COMMISSION OF THE EUROPEAN COMMUNITIES COM(94) 347, Brussels, 19.07.1994

[15] A. Binczewski, C. Mazurek, N. Meyer, J. Nabrzyski, S. Niwiñski, M. Stroiñski, Information Infrastructure of the Poznañ Science Society, Proceedings of EUNIS 1997

[16] M.Borkowski, Civil society needs and expectations: What does NGI do for the people?, The New Generation Internet Workshop, Poznań, 8.06.2017r

[17] http://www.poznan.pl/mim/api/dlaczego-otwieramyzasoby,p,25877,38303,38318.html (in polish)

[18] Garcia Robles, Ana, et al. "Introducing enoll and its living lab community." (2016). Poznan FutureLab, http://www.futurelab.p1/.

[19] Socientize Consortium. Green paper on citizen science. Citizen Science for Europe. Towards a better society of empowered citizens and enhanced research. Brussels. 2013.

[20] Silibello, C., Calori, G., Brusasca, G., Giudici, A., Angelino, E., Fossati, G., Peroni, E. and Buganza, E., 2008. Modelling of PM10 concentrations over Milano urban area using two aerosol modules. Environmental Modelling \& Software, 23(3), pp.333-343.

[21] Mazurek, C., Parkoła, T., \& Werla, M. (2013). Tools for mass digitization and long-term preservation in cultural heritage institutions. Review of the National Center for Digitization, (22), 75-80.

[22] Parkoła T. Masowa digitalizacja obiektów z wykorzystaniem pakietu oprogramowania DInGO. Biuletyn EBIB. 2015 Nov 12(162):1-7. https://dingo.psnc.pl/

[23] Dudczak, A., Heliński, M., Mazurek, C., Parkoła, T. and Werla, M., 2007. dLibra-platforma do budowy repozytoriów cyfrowych. Poznan: Wydawnictwo Politechniki Poznanskiej, pp.458-467.

[24] Werla M. (2017) Cloud technologies as a solution for digital collections management in small libraries. Paper presented at: IFLA WLIC 2017 - Wrocław, Poland Libraries. Solidarity. Society. in Session 150 - Preservation and Conservation, Acquisition and Collection Development.

[25] Gonçalves $\mathrm{P}$ et al. VRE Architecture and Interfaces Definition [Online] available at: http://ever-est.eu/wpcontent/uploads/EVER-EST DEL WP5-D5.1.pdf 